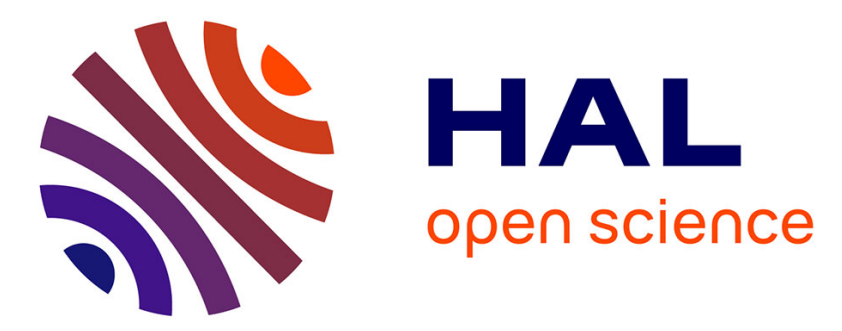

\title{
Les sciences naturelles en Bretagne, ou la réécriture de soi
}

Azélie Fayolle

\section{To cite this version:}

Azélie Fayolle. Les sciences naturelles en Bretagne, ou la réécriture de soi. Études Renaniennes, 2016, Renan personnage de roman, 117, pp.85 - 98. 10.3406/renan.2016.1649 . hal-03517631

\section{HAL Id: hal-03517631 \\ https://hal.science/hal-03517631}

Submitted on 7 Jan 2022

HAL is a multi-disciplinary open access archive for the deposit and dissemination of scientific research documents, whether they are published or not. The documents may come from teaching and research institutions in France or abroad, or from public or private research centers.
L'archive ouverte pluridisciplinaire HAL, est destinée au dépôt et à la diffusion de documents scientifiques de niveau recherche, publiés ou non, émanant des établissements d'enseignement et de recherche français ou étrangers, des laboratoires publics ou privés.

\section{(이) $\$$}

Distributed under a Creative Commons Attribution - NonCommercial - NoDerivatives| 4.0 


\section{Les sciences naturelles en Bretagne, ou la réécriture de soi}

\section{Azélie Fayolle}

\section{Résumé}

Loin des légendes primitives décrites dans les Souvenirs d'enfance et de jeunesse, la Bretagne de Renan a été un premier terreau pour le jeune séminariste. Par sa formation scolaire, comme par le milieu exceptionnel dont il a bénéficié, le jeune homme détrompe le tableau peint dans sa vieillesse : les sciences trouvent bien leur place, certes modeste, au collège de Tréguier. Les sciences naturelles sont surtout favorisées par l'entourage familial ; si ce premier cercle ne comporte aucun naturaliste, 1 amie d'Henriette, Sophie Ulliac-Trémadeure, a été une vulgarisatrice d'histoire naturelle. Bien plus, il est avéré que Renan a été abonné au Musée des familles, et a été amené à lire l'étonnante leçon transformiste de Pierre Boitard, Paris avant les hommes. Ainsi, quelques retouches historiennes apportent une nouvelle perspective à l'écriture autobiographique, qui permet à Renan une redéfinition de soi en homo novus.

\section{Citer ce document / Cite this document :}

Fayolle Azélie. Les sciences naturelles en Bretagne, ou la réécriture de soi. In: Études Renaniennes, N¹17, décembre 2016. Renan personnage de roman. pp. 85-98;

doi : https://doi.org/10.3406/renan.2016.1649

https://www.persee.fr/doc/renan_0046-2659_2016_num_117_1_1649

Fichier pdf généré le 09/05/2020 


\title{
LES SCIENCES NATURELlES EN BRETAGNE, OU LA RÉÉCRITURE DE SOI
}

\author{
J'étais fait en arrivant à Paris; avant de quitter la Bretagne, \\ ma vie était écrite d'avance'.
}

Renan dans ses Souvenirs d'enfance et de jeunesse évoque, dans un passage rapide mais célèbre, la possibilité avortée d'un autre avenir. Il aurait en effet pu poursuivre ses études en sciences naturelles et en physiologie:

Certes, je regrette maintenant que je n'aie point suivi son impulsion. Je serais sorti du séminaire sans avoir fait d'hébreu ni de théologie. La physiologie et les sciences naturelles m'auraient entrainé; or, je peux bien le dire, l'ardeur extrême que ces sciences vitales excitaient dans mon esprit me fait croire que, si je les avais cultivées d'une façon suivie, je fusse arrivé à plusieurs des résultats de Darwin que j'entrevoyais ${ }^{2}$.

Cette affirmation, déjà présente dans la non moins célèbre lettre à Marcellin Berthelot "Les sciences naturelles et les sciences historiques ", suit de peu le portrait haut en couleur de l'abbé Pinault, professeur de physique et de sciences naturelles de Renan au séminaire d'Issy, et la mention de l'importance qu'ont eu les cours pour le jeune élève:

Ce que M. Pinault m'apprit d'histoire naturelle générale et de physiologie m'initia aux lois de la vie. J'aperçus l'insuffisance de ce qu'on appelle le spiritualisme; les preuves cartésiennes de l'existence d'une âme distincte du corps me parurent toujours très faibles; dès lors, j'étais idéaliste, et non spiritualiste, dans le sens qu'on donne à ce mot $^{3}$.

Souvenirs d'Enfance et de Jeunesse, Paris, Calmann-Lévy, 1883, repris dans O.C., t. II, p. 760 .

2 Ibid., p. 851.

3 Ibid., p. 846. 
Le cours de sciences naturelles de l'abbé Pinault est ainsi présenté comme un moment inaugural, une véritable initiation. La découverte des sciences naturelles serait, d'après le Renan des Souvenirs, l'apanage d'Issy et de l'abbé Pinault. Renan renforce alors le contraste fort entre la Bretagne et l'arrivée à Paris:

Je me rappelle ce retour comme si c'était hier. Il y avait une lieue à faire à pied à travers la campagne. Les sonneries pieuses de l'Angélus du soir, se répondant de paroisse en paroisse, versaient dans l'air quelque chose de calme, de doux et de mélancolique, image de la vie que j'allais quitter pour toujours. Le lendemain, je partais pour Paris; le 7, je vis des choses aussi nouvelles pour moi que si j'avais été jeté brusquement en France de Tahiti ou de Tombouctou4.

L'arrivée à Paris signe l'entrée dans un monde nouveau. Les portraits des professeurs bretons et de leurs homologues parisiens, qu'ils soient d'Issy, de Saint-Sulpice ou de Saint-Nicolas, contrastent alors du tout au tout: pas de galerie de portraits pour les Bretons comme pour les Parisiens, alors que l'énigmatique bonhomme Système se voit consacrer un chapitre. Les portraits de ces "bons maitres " de Bretagne se font d'un bloc, dans un collectif anonyme, rappelant cette inscription dans des " temps primitifs" qui caractérise l'enfance " celte ", parfois mâtinée de gascon. La première formation scolaire du jeune Renan relève d'abord d'un fonds légendaire et d'une éducation humaniste, détachée du siècle:

J'ai raconté comment je reçus mon éducation dans un petit collège d'excellents prêtres, qui m'apprirent le latin à l'ancienne manière (c'était la bonne), c'est-à-dire avec des livres élémentaires détestables, sans méthode, presque sans grammaire, comme l'ont appris, au $\mathrm{Xv}^{e}$ et au $\mathrm{XVI}^{\mathrm{e}}$ siècles, Érasme et les humanistes qui, depuis l'antiquité, l'ont le mieux su'.

Le passage de Tréguier à Paris est plus qu'un passage de la province reculée à la capitale: il prend toutes les formes d'un saut dans le temps. Renan, quand il se fait historien de lui-même, joue de

$\begin{array}{ll}{ }^{4} & \text { Ibid., p. } 807 . \\ 5 & \text { Ibid., p. } 787 .\end{array}$ 
contrastes forts, et affirme la nullité de sa première éducation, au moins pour ce qui ressort des sciences:

Les études d'histoire et de sciences naturelles étaient également nulles. En revanche, on nous faisait pousser assez loin l'étude des mathématiques. J'y apportais une extrême passion; ces combinaisons abstraites me faisaient rêver jour et nuit ${ }^{6}$.

Cette opposition entre enfance et jeunesse se trouve néanmoins estompée par un examen attentif de sa correspondance et des détails de sa formation bretonne. Si, comme l'affirme René d'Ys, la période bretonne de Renan reste la " moins connue" ", elle est aussi un point aveugle des études qui lui sont consacrées: tout laisse penser que la formation scientifique de Renan débute au séminaire d'Issy, à Paris. La correspondance reste, certes, allusive; la mention des cours ne donne pas lieu à développements dans les lettres. Ce sont d'ailleurs plutôt les cours de physique qui y sont régulièrement évoqués. Il ne s'agit pas ici de voir si les sciences naturelles ont été pour le jeune sulpicien un plus ou moins grand choc par rapport à la physique, ni de relativiser l'importance de ces cours sur la formation intellectuelle et spirituelle du jeune Renan; ni de voir si, éventuellement, Renan aurait pu être Darwin. Il s'agit de confronter l'écriture de soi du Renan des Souvenirs à celle du Renan jeune; de vérifier les pistes ainsi tracées, qui permettent non seulement une reconstitution de la première formation scientifique de Renan, mais également de saisir quelques traits de son écriture autobiographique.

La formation scientifique de Renan a bien commencé dès la Bretagne. Sa formation primaire n'inclut certes pas de cours de sciences: à l'école des Frères de Lannion de 1828 à 1830, puis à celle des Frères de Lamennais jusqu'en 1832, Renan n'apprend, conformément au programme de Jean-Marie Lamennais, qu'à lire, écrire et compter, dans un emploi du temps qui laisse une large place aux cours de catéchisme. Il a peut-être pu suivre des cours optionnels de dessin linéaire à Tréguier, mais rien ne l'atteste. Si les

Ibid., p. 732.

7 René d'Ys, Renan en Bretagne, d'après des documents nouveaux, Paris, Émile-Paul éditeur, 1904 , p. 112. 
programmes promulgués par la loi Guizot de 1833 instaurent un enseignement scientifique dès le secondaire, ils ne trouvent qu'une application limitée, surtout en province. Le statut ecclésiastique du collège de Tréguier ne le contraint pas autant qu'un établissement laïque à l'application de ces programmes et rend d'autant plus ardue la connaissance du contenu des cours. Par ailleurs, Renan lui-même ne correspondait pas alors régulièrement avec sa famille, puisqu'il vivait avec sa mère, et peu de lettres de cette période sont conservées. Ce n'est que par de multiples croisements qu'il est possible de restituer non seulement la formation scolaire, mais aussi le bain culturel scientifique dans lequel Ernest Renan évoluait en Bretagne. Cette reconstitution est à la fois l'esquisse d'une formation scolaire, mais également une première étude de la culture et des réseaux du jeune Renan, ainsi que de l'influence, bien connue mais encore sousestimée, de sa sœur Henriette.

Henriette part pour Paris en 1835, où elle rejoint son frère Alain, qui déménage la même année à Saint-Malo; ce sont d'abord les déplacements des membres de la famille et les quelques lettres les plus anciennes conservées qui permettent d'obtenir de précieux renseignements sur la formation de Renan. Ce sont ensuite les lettres envoyées par le jeune séminariste depuis Paris, qui permettent d'obtenir quelques indices supplémentaires.

Le petit séminaire de Tréguier semble avoir offert une formation des plus satisfaisantes, en tout cas à son élève le plus célèbre, Ernest Renan, qui y a été scolarisé de 1832 à 1838. Ses Souvenirs d'enfance et de jeunesse marquent toute sa gratitude pour ses professeurs et évoquent rapidement la nullité des "études d'histoire et de sciences naturelles ${ }^{8}$. Les bulletins de Renan ont été conservés et publiés", mais ceux-ci ne signalent pas de sciences naturelles au programme du petit séminaire. Plus diserte, la biographie bretonne de Renan signale l'intérêt du chanoine Auffret pour les sciences physiques ${ }^{10}$. Les petits séminaires suivent alors les grands axes des programmes des collèges laïques, mais la situation de ces collèges comme celle des

\footnotetext{
Ibid., p. 164.
}

9 Théophile Janvrais, "Ernest Renan écolier (ses notes de classe - ses palmarès) ", in Annales de Bretagne, t. 19, $\mathrm{n}^{\circ} 1,1903$.

10 René d'Ys, Renan en Bretagne..., op. cit., p. 118. 
séminaires reste disparate sur l'ensemble du territoire français. Bien plus, les sciences physiques peuvent inclure des éléments d'histoire naturelle sans que ceux-ci soient mentionnés dans l'intitulé des cours.

S’il est impossible d'avoir des précisions sur les cours suivis, il reste néanmoins attesté que Renan a eu des cours de sciences dès sa scolarité au petit séminaire de Tréguier, ce qui est plus significatif que le simple intérêt de l'abbé Auffret. Depuis Paris, Ernest Renan n'oublie pas en effet ses professeurs trégorrois, et charge sa mère de les saluer:

Faites de même mes compliments à $M$. Gouriou, que je suis bien fâché de n'avoir pas vu avant mon départ, au bon monsieur Potier, à Monsieur Brouster, et particulièrement à Monsieur Delangle. Dites à ce bon monsieur qui me portait tant d'intérêt que je n'oublierai jamais tout ce qu'il a fait pour $\mathrm{moi}^{11}$.

L'abbé Delangle, indique une note de Jean Balcou, est un " professeur de sciences qui réorganisa le collège 12 ". Là encore, le détail des cours suivis serait riche d'informations pour la connaissance du jeune élève, mais reste, pour le moment, impossible à établir. L'attachement de Renan pour ce professeur est réaffirmé dans deux autres lettres conservées, dont l'une est éditée pour la première fois dans la Correspondance générale $e^{13}$. Ce goût précoce pour les mathématiques a été évoqué par Renan dans ses Souvenirs d'enfance et de jeunesse, mais cette appétence est plus large, comme le montre cette allusion dans une lettre de Manon Renan à ses enfants, restée elle aussi inédite avant la Correspondance générale:

Dépêchez-vous d'arriver, mes enfants, détournez votre frère de ses auteurs de mathématiques, surtout de physique, il est fou de tout cela, je ne puis l'empêcher de travailler ${ }^{14}$.

11 Ernest Renan, letrre à sa mère, 8 septembre 1838, Correspondance Générale, t. I, 18361845, textes réunis, classés et annotés par Jean Balcou, Paris, Honoré Champion, 1995. p. 55.

12 Ibid., note 3.

13 Lettre d'Ernest Renan à sa mère, 26 décembre 1838, 10 janvier 1839, ibid., p. 77-82.

14 Lettre d'Ernest Renan à Alain, au verso, lettre de sa mère à Alain, 27 août 1838, ibid., p. 47. 
Ce goût est entretenu par sa famille, comme le signale le regret du jeune séminariste de ne pouvoir s'entretenir de physique avec son frère aîné Alain. Il reste délicat de trop pousser suppositions et spéculations, mais il apparaît clairement que la formation de Renan en sciences expérimentales débute dès sa jeunesse trégorroise, par sa formation scolaire au Collège ecclésiastique, et grâce à un milieu familial éclairé et favorable. Il saura d'ailleurs s'en rappeler durant ses années parisiennes: il peut ainsi convoquer ses connaissances physiques, comme lorsqu'il évoque un " phénomène acoustique " qui l'étonne lors d'une visite du Panthéon, et dont il " réserve les détails [...] pour le physicien Guyomard et pour le cher Liard qui est aussi physicien en herbe ${ }^{15}$ ". Cette connivence affichée avec ses amis bretons, qui le rejoindront l'année suivante à Paris, montre bien le partage de connaissances communes acquises en cours, certainement sous l'égide de l'abbé Auffret.

Complétant cette première approche scientifique, l'influence qu'a eue Henriette, "l'une des plus émouvantes et plus fortes figures d'intellectuelles au XIX ${ }^{e}$ siècle $^{16}$ » sur son frère cadet, est bien connue; son rôle sur la formation en sciences naturelles de Renan est, là aussi, primordial. Éduquée à Lannion par une " personne distinguée par le goût et les manières ", l'aînée des Renan est " très instruite ${ }^{17}$ ". Son goût pour l'étude se révèle par sa profession d'institutrice, d'abord en Bretagne, puis dans une pension parisienne. Bien qu' elle soit titulaire d'un brevet de capacité pour l'enseignement primaire, ce diplôme ne permet pas de connaître précisément ses compétences en sciences naturelles, exigées pour les hommes mais non pour les femmes. Le contrat signé avec les Zamoyski ne donne pas plus d'informations ${ }^{18}$. Mais Henriette Renan a montré un intérêt pour cette discipline: sa collaboration au Journal des Jeunes Personnes, l'amène à rédiger l'article

15 Lettre d'Ernest Renan à sa mère, 10 janvier 1839, ibid., p. 80.

16 Jean Balcou, Renan et la Bretagne, Paris, Honoré Champion, 1992, p. 16.

17 René d'Ys, Renan en Bretagne..., op. cit., p. 103.

18 Contrat signé par Henriette et les banquiers parisiens du comte, les frères Mallet, le 11 janvier 1841 ; voir Eva Stankovitch, Henriette Renan. Sa carrière d'enseignante, thèse de doctorat de littérature française présentée sous la direction de Jean Balcou, U.B.O. Brest, 1993, cité p. 37. 
"Lectures choisies. Astronomie et météorologie " en juillet 1848 ", et il semble, qu'en Pologne elle lise régulièrement les CEuvres posthumes de Bernardin de Saint-Pierre ${ }^{20}$. Elle signale en outre une visite rapide des " premières allées " au Jardin des Plantes à son frère, le 6 décembre $1837^{21}$.

Le soin qu'Henriette voue à son frère dès son plus jeune âge a une importance directe non seulement sur les résultats scolaires et la formation de son cadet, mais il élargit également son horizon culturel. Ses conseils émaillent ses lettres à sa famille, dont elle s'est éloignée pour enseigner à Paris. Ce soin est également matériel et livresque: point important, elle indique ainsi, dans une lettre à sa mère du 29 septembre 1836, réaliser " que l'abonnement d'Ernest au Musée des familles expire ce $1^{\text {er }}$ octobre ". Ce passage de la correspondance familiale indique que, grâce à sa sœur, Ernest Renan a été abonné au moins depuis le $1^{\text {er }}$ octobre 1835 à cette revue mensuelle populaire ${ }^{22}$. Le Musée des familles. Lectures du soir a pour but de " rendre la littérature populaire ${ }^{23}$ " et diffuse une culture généraliste, avec des textes de littérature comme de vulgarisation scientifique. Chaque numéro présente trois parties, un Magazine, regroupant des curiosités, des Voyages, et surtout une Revue, partie principale du Musée des familles qui rassemble « des études historiques, des études de mœurs et des études d'histoire naturelle ${ }^{24}$ ». On trouve dans ce volume des études d'histoire naturelle accompagnées de gravures, sur différents animaux (ours, singes, crocodiles, jaguar,

19 Ibid., on trouve la liste des articles d'Henriette Renan pour cette revue p. 117 et suivantes. Cette liste doit être élargie, la plupart de ses articles étant parus anonymement, comme le signale Renan dans Ma saur Henriette.

20 La liste des lectures d'Henriette Renan est établie par Eva Stankovitch à partir des citations et extraits présents dans ses lettres.

21 Lettre d'Henriette Renan à Ernest Renan, 6 décembre 1837, C.G. I, op. cit., p. 43.

22 Il est pour le moment impossible de savoir si l'abonnement du jeune Renan remonte à plus d'une année; le Musée des familles a été lancé le $1^{\text {cr }}$ octobre 1833 , après une grande campagne de publicité à Paris, et ne propose pas de vente au numéro, mais uniquement des souscriptions d'une année. La date annoncée pour le renouvellement permet de supposer un abonnement dès les premiers numéros de la revue. Ce ne serait pas alors Henriette, mais Alain qui aurait inscrit le jeune garçon.

23 Le Musée des familles, vol. 3, 1835-1836, [en ligne], [consulté le 26/02/2016], p. 378. URL: http://gallica.bnf.fr/ark:/12148/bpt6k5401606t 
oiseaux, etc.), des études sur les curiosités naturelles, l'éthologie animale, toutes signées du naturaliste Pierre Boitard. La plupart se découpent en une présentation de l'espèce et de ses parents, de leur habitat et habitudes, poursuivie par quelques souvenirs personnels ou anecdotes. Ces leçons d'histoire naturelle couplent des questions de zoologie (la morphologie des animaux y occupe une place importante) ainsi que de phylogenèse (à l'époque, essentiellement la classification des espèces, plus que leur histoire, y est abordée. Pierre Boitard livre ainsi des textes scientifiquement aboutis, entrecoupés de réflexions personnelles et de petits récits qui en agrémentent la lecture, comme les planches, le plus souvent pour l'année 1835-36 de Susemilh, graveur du Jardin des Plantes). Parmi ces leçons s'en détache une qui, gardant la même présentation scientifique, présente un rêve du naturaliste. Ce très étonnant "Paris avant les hommes", qui affiche une magistrale gravure de ptérodactyle à sa tête, est très certainement une première mouture du roman posthume de Boitard Paris avant les hommes (1861), considéré comme la première fiction préhistorique mondiale. Ce texte, daté de juin 1836, ne peut qu'avoir été lu par le jeune Renan, dont le sérieux et l'assiduité sont notoires: il est difficile de ne pas l'imaginer, curieux et attentif, penché sur ces lectures offertes par sa sœur, malgré les difficultés économiques de la famille.

$\mathrm{Si}$ « Paris avant les hommes " peut sembler une œuvre mineure issue d'une revue aujourd'hui oubliée, un examen plus attentif en dévoile toute la singularité. L'ensemble du texte reprend le dispositif du Diable boiteux de Lesage: le naturaliste, grâce au diable boiteux, est transporté dans le temps, et voit la transformation de la terre et des êtres. La reconstitution des âges en six périodes donne le cadre général de la formation terrestre, et concorde avec le récit biblique de la Genèse. Si le récit se place sous le patronage explicite de Cuvier, allant jusqu'à préciser dans une note infrapaginale une légère divergence avec le grand maître pour le détail d'une oreille, le déroulement du texte dans son ensemble est résolument anticuviériste et, contre toute attente, transformiste.

L'ensemble de ces tableaux naturalistes est présenté comme un rêve du naturaliste, qui, une fois réveillé, justifie et démontre ces reconstitutions: les détails du texte sont ainsi, a posteriori, justifiés 
scientifiquement à partir des dernières découvertes et théories des savants (mais Lamarck n'est pas cité, pas plus que d'autres savants non cuviéristes). Cette justification scientifique de la rêverie naturaliste peut rappeler, anachroniquement, le dispositif de la Vie de Jésus, qui oscille entre les attestations philologiques et le plaisir évocateur de la pastorale; la postface du texte encadre le matériau onirique, en ramenant la fiction à une hypothèse probable, matériau historique constant de Renan devenu lui-même un savant. "Paris avant les hommes " montre surtout un contact précoce de Renan avec le transformisme. On retrouve par ailleurs dans ce texte comme dans d'autres leçons de Pierre Boitard quelques images fortes qui reviendront dans ses écrits, comme celle de l'animal-plante. Ce contact précoce avec le transformisme est de plus significatif en ce qu'il amène une nouvelle conception du temps: en refusant le catastrophisme de Cuvier, Boitard plaide pour une évolution lente et un temps long:

La création est lente, elle marche pas à pas; mais elle est uniforme par toute la terre, parce qu'elle est soumise à une règle uniforme et invariable, sans laquelle elle est impossible. Cette règle consiste à procéder du simple au composé, d'abord double, puis triple, puis quadruple, ainsi de suite jusqu'à l'organisation la plus compliquée $e^{25}$.

L'uniformitarisme et la lenteur des évolutions contredisent la théorie des catastrophes locales et " révolutions du globe " de Cuvier, et conditionnent une histoire de l'organisation des êtres. Cette organisation, qui se fait du simple au complexe est celle qui, repensée, donne le passage de la synthèse à l'analyse tant dans les langues que pour les religions, comme le montrent les travaux ultérieurs de Renan. Si Boitard pense un mouvement analytique, quand Renan préférera penser une complexité originelle, ce sont bien des réflexions structurant sa pensée qui paraissent trouver ici un point d'origine. Par l'affirmation d'une évolution uniformitariste, opposée au catastrophisme cuviériste, Pierre Boitard se détache résolument, dans une revue populaire et à grande diffusion, des théories admises et reconnues, et postule une évolution des êtres vivants: 
Ne savez-vous pas que les êtres sont modifiés en raison des climats et des milieux qu'ils habitent? Qui vous a dit que les palmiers de ce temps-là, habitant l'endroit où nous sommes, n'étaient pas organisés de manière à supporter sans inconvénient un froid de 12, de 20 degrés Réaumur? Qui vous oblige de croire que la terre a fait une cabriole sur son axe parce qu'elle aurait reçu en passant un coup de queue d'une comète? de faire du globe une boulette refroidie, de l'atmosphère un bain de vapeur, de la mer un consommé servi chaud, et autres billevesées de la même force ${ }^{26}$ ?

Le transformisme de Boitard se tourne résolument vers une théorie des milicux, dont les grands linéaments pourraient se trouver dans la théorie des climats de Montesquieu. Adaptée aux êtres vivants et non plus à l'esprit des peuples, cette adaptation des organismes à leur environnement est bien lamarckienne: les êtres vivants développent tel ou tel caractère en raison de leur milieu naturel.

Avant même son arrivée à Saint-Nicolas, Renan a ainsi bien eu une formation en sciences naturelles qui, si elle s'ajoute à d'éventuels cours de collège, est en tout cas, grâce au Musée des familles, non seulement congruente avec les grandes problématiques de la première moitié du $\mathrm{XIX}^{\mathrm{e}}$ siècle, comme pour les questions de classification des espèces, mais également non académique, et potentiellement subversive et irréligieuse. Pour le futur penseur du fieri, cette mise en mouvement spectaculaire, et iconographique, de la nature à un jeune âge a pu jouer un rôle déterminant dans l'élaboration de sa pensée; elle montre déjà l'importance précoce, jusqu'ici sous-évaluée, des sciences de la nature dans la formation bretonne de Renan.

Henriette Renan ne se contente d'ailleurs pas de conseiller son frère et de l'abonner au Musée des familles. Elle lui permet également d'évoluer, par son intermédiaire, dans un climat scientifique avant même son arrivée à Paris, climat qui lui sera particulièrement favorable une fois dans la capitale. Il est ardu d'établir l'ensemble des relations d'Henriette Renan: celles-ci sont encore peu documentées. Il reste possible d'entrevoir l'étendue de ce réseau par quelques passages conservés de sa correspondance. Ainsi, Henriette révèle à sa mère qu'elle a pu bénéficier, grâce à ses relations, d'un traitement

26 Ibid., p. 260. 
de faveur au moment de passer ses examens grâce à $M$. Guichard et surtout " un de ses amis" ${ }^{27}$ ". Il est bien difficile de savoir qui est cet " ami "; il n'en reste pas moins que de telles facilités accordées à Henriette Renan sont riches d'enseignements: elles garantissent en effet que la jeune femme bénéficie à Paris d'un réseau de grande qualité, notamment chez les savants, et qu'elle est de plus reconnue pour ses qualités qui l'exemptent des examens les plus scolaires. Ces qualités sont réaffirmées par le succès d'Henriette à ses examens.

Elle rencontre aussi $M^{11 \mathrm{le}}$ Ulliac $^{28}$ en 1837 , qui deviendra une de ses meilleures amies. Sophie Ulliac-Trémadeure, femme de lettres, qui collabore puis dirige le Journal des Jeunes Personnes. La meilleure amie parisienne d'Henriette reste aujourd'hui méconnue, mais elle est une figure marquante de la diffusion scientifique, notamment de l'histoire naturelle, attentive au positivisme ${ }^{29}$ comme à la condition féminine.

Lors de la rencontre des deux femmes en 1837, Sophie Ulliac a déjà signé des Contes aux jeunes Agronomes, aux jeunes Artistes, aux jeunes Naturalistes. Reconnue comme " auteur moral ", ses ouvrages de vulgarisation régulièrement réédités bénéficient d'une bonne diffusion en milieu scolaire. Si Léon Dubreuil note qu'entre 1837 et 1845, "elle s'intéresse surtout à l'éducation morale des jeunes filles et à l'histoire naturelle ${ }^{30}$ ", il faut faire remonter cet intérêt d'au moins deux années avant la rencontre d'Henriette, et constater qu'il ne s'interrompt pas: en plus du cours Quelques legons d'histoire naturelle qu'elle donne à partir de 1835 dans le Journal des Jeunes Personnes, elle livre des recensions d'ouvrages scientifiques à divers journaux, et poursuit son ouvre de vulgarisation des sciences naturelles en volumes. La riche production de Sophie Ulliac laisse ainsi penser que sa future collaboratrice et amie a pu voir naître, ou approfondir, un intérêt pour les sciences naturelles, spéculation qui laisse à penser

\footnotetext{
27 Henriette Renan à sa mère, 16 janvier 1837, C.G. I, op. cit., p. 36.

28 Sur $M^{\text {lle }}$ Ulliac, voir les articles de Léon Dubreuil: "Une amie d'Henriette Renan, Sophie Ulliac-Trémadeure ", in Annales de Bretagne, n 66-2, 1959, et Isabelle Pannier, "Sophie Ulliac Trémadeure: les contradictions de la vertu ", in Romantisme, $\mathrm{n}^{\circ} 77$, 1992, p. 33-36.

29. Elle a fait un compte rendu du cours d'Auguste Comte dans la revue De l'Éducation positive en 1835.

30 Léon Dubreuil, "Une amie d'Henriette Renan... ", op. cit., p. 218.
} 
que l'intérêt de Renan lui-même pour les sciences a pu être quelque peu antérieur à son arrivée à Paris. Ernest Renan aurait-il eu des livres de $M^{\text {lle }}$ Ulliac? Sans que cela soit attesté, la chose n'est pas impossible. Les relations entre Sophie Ulliac et Ernest Renan sont bien éloignées de l'amitié qui lie les deux femmes, mais elles existent: Sophie Ulliac reste une aide et un soutien pour Ernest Renan lors du long séjour de sa sœur en Pologne, et elle garde l'honneur d'avoir été la première à publier ses premiers textes, deux énigmes historiques, véritable corvée pour le jeune homme.

Autre soutien d'importance, l'ami qui a fait connaitre le palmarès d'Ernest Renan à Félix Dupanloup, responsable du petit séminaire de Saint-Nicolas, n'est autre que le médecin Jean-BaptisteFélix Descuret. Il est mentionné dans une lettre du 31 août 1838 d'Henriette à Renan ${ }^{31}$, et est identifié par une note de Jean Balcou. J.-B. Félix Descuret est en effet le médecin d'Henriette, qu'elle a rencontré dans la pension où ils travaillent. Renan le rencontre dès le lendemain de son arrivée à Paris. J.-B. Félix Descuret est également auteur et éditeur scientifique, et a participé à l'édition des œuvres complètes de Jacques Delille. C'est aussi probablement lui qui a provoqué la rencontre avec Daremberg, autre médecin qui a accompagné Renan lors de sa mission en Italie.

Ernest Renan a donc eu des cours scientifiques à Tréguier, un environnement favorable à une première formation en sciences naturelles, et ce, essentiellement grâce à sa sœur: abonné au moins depuis octobre 1835 au Musée des familles, le jeune séminariste a eu accès à des leçons d'histoire naturelle mensuelles régulières, à des gravures exécutées par des naturalistes et à l'évolutionnisme; le travail d'Henriette Renan comme ses relations, ont offert au jeune garçon une première formation en sciences naturelles, et lui ont permis d'entrer dans des réseaux de médecins, vulgarisateurs et déjà, certainement, des savants. À ce titre, l'accueil fait au jeune Trégorrois par le médecin Descuret dès le lendemain de son arrivée inaugure superbement l'importance des sciences naturelles pour Renan avant les cours d'Issy, puis la rencontre de Marcellin Berthelot.

31 Lettre d'Henriette Renan à Ernest Renan, 31 août 1838, C.G. I, op. cit., p. 53. 
Ainsi, le contraste saisissant entre la Bretagne et Paris présenté par les Souvenirs est à nuancer: sa formation scientifique, bien qu'insuffisante, n'a pas débuté avec les cours de l'abbé Pinault. Plus qu'un portrait de soi, c'est bien un portrait par paysages, par tableaux que propose la célèbre autobiographie. Infidèles, évocateurs, ces souvenirs se retrouvent sous le signe de l'hircocerf, ici amputé d'Henriette, qui en est la grande absente. Si ce qu'on dit de soi est toujours "poésie ", comme il l'annonce lui-même dans la préface de ses Souvenirs, c'est une poésie du contraste et de l'homo novus nouveau qui apparait ainsi. Renan s'offre comme un homme nouveau s'étant lui-même formé grâce à de prestigieux mentors, comme l'abbé Pinault, mais sans femmes. La réalité que l'on parvient à esquisser est plus nuancée; la Bretagne légendaire et primitive est en fait, pour part, déjà parisienne, notamment grâce à sa sœur, la grande absente des Souvenirs, et déjà nourrie par les sciences, physiques comme naturelles.

Ce modeste travail sur la formation en sciences naturelles de Renan avant son arrivée à Paris est un premier pas dans une étude plus vaste; la thèse dans laquelle il s'inclura vise à comprendre les liens, affichés, entre la pensée de l'histoire et les sciences de la nature pour Renan.

Entre les sciences naturelles et les sciences historiques, Ernest Renan n'a pas seulement fait un choix de carrière: philologue, historien et philosophe, il se situe au croisement de ces disciplines, non seulement par ses réseaux amicaux et scientifiques, mais aussi par sa connaissance précoce des théories évolutionnistes. Le projet historique se nourrit en effet, dès ses Cabiers de jeunesse, de l'histoire naturelle, et esquisse déjà sa philosophie du fieri, dont l'Avenir de la science porte la marque. L'empreinte des sciences naturelles s'exhibe dans L'Histoire des origines du christianisme, pensée comme une embryogénie de l'esprit humain; elle reste présente dans l'ensemble de son œuvre.

Le projet de cette thèse, intitulée Ernest Renan: savoirs de la nature et pensée historique, vise à retracer ce parcours intellectuel dans l'ensemble des dimensions de ce transfert disciplinaire: historique, quand il s'agit de connaître la formation scolaire et intellectuelle d'Ernest Renan, de sa scolarité bretonne aux séminaires parisiens et 
à l'agrégation de philosophie; littéraire, pour analyser un ensemble de métaphores et d'images issues des sciences naturelles nourrissant son œuvre; c'est ainsi que le germe biblique se fait scientifique, ou que l'image de l'organisme guide explicitement la rédaction. La Vie de Jésus n'étant jamais qu'une réécriture, ce sont également les outils de la narratologie et de l'intertextualité qui permettront de spécifier l'écriture historienne de Renan. C'est sur une perspective épistémologique et philosophique que débouchera ce travail, qui vise à dégager les nouvelles modalités du texte historique pour Renan, et sur quelle nouvelle définition de la Science et de la division disciplinaire des sciences, aboutit le travail de Renan. C'est en effet en fondant son Histoire du christianisme comme une embryogénie que Renan fonde l'histoire comme science historique; c'est une même pensée du temps qui se retrouve dans l'évolution des espèces et du globe comme dans la transformation des langues et des religions. Ce travail doit d'abord être historique, en retraçant les étapes de la formation et les réseaux du savant, mais sera essentiellement littéraire: la pensée analogique de Renan nourrit sa prose de métaphores et d'images dont il s'agit de retracer le parcours, du germe, biblique et biologique, de l'organisme, modèle méthodologique et esthétique, à l'épidémiologie, qui modélise les transferts culturels et la diffusion des langues comme des religions. C'est ainsi un nouveau statut du texte historique et du document qui se nourrit de cette hybridation générique, comme une nouvelle épistémologie fondée par le croisement d'une imagerie scientifique et de fictions historiques.

\author{
Azélie FAYOLLE ${ }^{32}$ \\ (biolographe, Région Île de France)
}

\footnotetext{
Azélie Fayolle prépare une thèse incitulée Ernest Renan: savoirs de la nature et pensée historique, sous la direction de Gisèle Séginger (UPEM, LISAA EA 4120, membre de l'IUF).
} 